\title{
Total domination on anti fuzzy graph
}

\author{
R.Muthuraj ${ }^{1}$ and A.Sasireka ${ }^{2}$ \\ ${ }^{1}$ Department of Mathematics, H.H.The Rajah's College, Pudukkottai-622 001, TamilNadu, India \\ ${ }^{2}$ Department of Mathematics, PSNA College of Engineering and Technology, Dindigul-624 622, TamilNadu, India
}

Received: 2 April 2018, Accepted: 10 September 2018

Published online: 25 October 2018.

\begin{abstract}
In this paper, the concept of total dominating set and total domination number on anti fuzzy graph is introduced. The bounds on total domination number of an anti fuzzy graph are obtained. Using strong adjacency matrix, an algorithm is derived for finding minimal total dominating set of anti fuzzy graph $\mathrm{G}_{A}$. These concepts are applied on anti cartesian product of anti fuzzy graphs and obtained the results on them.
\end{abstract}

Keywords: Anti fuzzy graph, total domination number, v-nodal anti fuzzy graph, uninodal anti fuzzy graph.

\section{Introduction}

Domination plays a vital role in graph theory. When vagueness occurs within objects and its relation, the concept of fuzzy graph is developed. Sometimes uncertainty occurs with only the relation between the objects; in such cases an anti fuzzy graph is introduced. R.Seethalakshmi and R.B.Gnanajothi[8] introduced the definition of anti fuzzy graph. R.Muthuraj and A.Sasireka[6] defined some types of anti fuzzy graph. Moderson J.N. and Peng[3] defined the cartesian product of two arbitrary fuzzy graphs. R.Muthuraj and A.Sasireka[5, 7] illustrated some operations on anti fuzzy graphs such as anti union, anti join, anti cartesian product and anti composition and also introduced the concept of domination on anti fuzzy graph. A.Somasundaram and S.Somasundaram[10] introduced total domination in fuzzy graphs using effective edges. In this paper, we introduce the concept of total domination number on anti fuzzy graph and obtained the bounds on them. Using strong neighbourhood fuzzy matrix, an algorithm is described to predict the total dominating set for an anti fuzzy graph. These concepts are applied on anti cartesian product of anti fuzzy graphs. The results are examined and some theorems are derived from them.

\section{Preliminaries}

In this section, basic concepts of anti fuzzy graph are discussed. Notations and more formal definitions which are followed as in $[4,5,6,9]$.

Definition 1. [9] An anti fuzzy graph $G_{A}=(\sigma, \mu)$ is a pair of functions $\sigma: V \rightarrow[0,1]$ and $\mu: V \times V \rightarrow[0,1]$, with $\mu(u, v) \geq$ $\sigma(u) \vee \sigma(v)$ for all $u, v \in V$.

Note. $\mu$ is considered as reflexive and symmetric. In all examples $\sigma$ is chosen suitably. i.e., undirected anti fuzzy graphs are only considered.

Definition 2. [5] The order $p$ and size $q$ of an anti fuzzy graph $G_{A}=(V, \sigma, \mu)$ are defined to be $p=\sum_{x \in V} \sigma(x)$ and $q=\sum_{x y \in E} \mu(x, y)$. It is denoted by $O(G)$ and $S(G)$. 
Definition 3. [6] Two vertices $u$ and $v$ in $G_{A}$ are called adjacent if $(1 / 2)[\sigma(u) \vee \sigma(v)] \leq \mu(u, v)$.

Definition 4. [4] The anti complement of anti fuzzy graph $G_{A}=(\sigma, \mu)$ is an anti fuzzy graph $\overline{G_{A}}=(\bar{\sigma}, \bar{\mu})$ where $\bar{\sigma}=$ $\sigma$ and $\bar{\mu}(u, v)=\mu(u, v)-(\sigma(u) \vee \sigma(v))$ for all $u, v$ in $V$.

Definition 5. [9] An anti fuzzy graph $G_{A}=(\sigma, \mu)$ is a strong anti fuzzy graph of $\mu(u, v)=\sigma(u) \vee \sigma(v)$ for all $(u, v) \in \mu^{*}$ and $G_{A}$ is a complete anti fuzzy graph if $\mu(u, v)=\sigma(u) \vee \sigma(v)$ for all $(u, v) \in \mu^{*}$ and $u, v \in \sigma^{*}$. Two vertices $u$ and $v$ are said to be neighbors if $\mu(u, v)>0$.

Definition 6. [6] An edge $e=\{u, v\}$ of an anti fuzzy graph $G_{A}$ is called an effective edge if $\mu(u, v)=\sigma(u) \vee \sigma(v)$.

Definition 7. [5] $u$ is a vertex in an anti fuzzy graph $G_{A}$ then $N(u)=\{v:(u, v)$ is an effective edge $\}$ is called the open neighborhood of $u$ and $N[u]=N(u) \cup\{u\}$ is called closed neighborhood of $u$.

Definition 8. [4] A path $P_{A}$ in an anti fuzzy graph is a sequence of distinct vertices $u_{0}, u_{1}, u_{2}, \ldots, u_{n}$ such that $\mu\left(u_{i-1}, u_{i}\right)>0,1 \leq i \leq n$. Here $n \geq 0$ is called the length of the path $P_{A}$. The consecutive pairs $\left(u_{i-1}, u_{i}\right)$ are called the edges of the path.

Definition 9. [4] A cycle in $G_{A}$ is said to be an anti fuzzy cycle if it contains more than one weakest edge. It is denoted by $C_{A}$.

Definition 10. [5] Let $G_{A}^{*}=G_{A_{1}}^{*} \times G_{A_{2}}^{*}=\left(V, E^{\prime}\right)$ be the anti cartesian product of anti fuzzy graphs where $V=V_{1} \times V_{2}$ and $\left.E^{\prime}=\left\{\left(u_{1}, u_{2}\right),\left(u_{1}, v_{2}\right) / u_{1} \in V_{1},\left(u_{2}, v_{2}\right) \in E_{2}\right\} \cup\left(u_{1}, w_{2}\right),\left(v_{1}, w_{2}\right) / w_{2} \in V_{2},\left(u_{1}, v_{1}\right) \in E_{1}\right\}$. Then the anti cartesian product of two anti fuzzy graphs, $G_{A}=G_{A_{1}} \times G_{A_{2}}:\left(\sigma_{1} \times \sigma_{2}, \mu_{1} \times \mu_{2}\right)$ is an anti fuzzy graph and is defined by

$\left(\sigma_{1} \times \sigma_{2}\right)\left(u_{1}, u_{2}\right)=\max \left\{\sigma_{1}\left(u_{1}\right), \sigma_{2}\left(u_{2}\right)\right\}$ for all $\left(u_{1}, u_{2}\right) \in V$,

$\left(\mu_{1} \times \mu_{2}\right)\left(\left(u_{1}, u_{2}\right),\left(u_{1}, v_{2}\right)\right)=\max \left\{\sigma_{1}\left(u_{1}\right), \mu_{2}\left(u_{2}, v_{2}\right)\right\}$ for all $u_{1} \in V_{1}$ and $\left(u_{2}, v_{2}\right) \in E_{2}$,

$\left(\mu_{1} \times \mu_{2}\right)\left(\left(u_{1}, w_{2}\right),\left(v_{1}, w_{2}\right)\right)=\max \left\{\sigma_{2}\left(w_{2}\right), \mu_{1}\left(u_{1}, v_{1}\right)\right\}$ for all $w_{2} \in V_{2}$ and $\left(u_{1}, v_{1}\right) \in E_{1}$.

Then the anti fuzzy graph $G_{A}=\left(\sigma_{1} \times \sigma_{2}, \mu_{1} \times \mu_{2}\right)$ is said to be the anti cartesian product of anti fuzzy graphs $G_{A_{1}}=\left(\sigma_{1}\right.$, $\left.\mu_{1}\right)$ and $G_{A_{2}}=\left(\sigma_{2}, \mu_{2}\right)$.

Definition 11. [6] Every vertex in an anti fuzzy graph $G_{A}$ has unique fuzzy values then $G_{A}$ is said to be v-nodal anti fuzzy graph. i.e. $\sigma(u)=c$ for all $u \in V\left(G_{A}\right)$.

Definition 12. [6] Every edge in an anti fuzzy graph $G_{A}$ has unique fuzzy values then $G_{A}$ is said to be e-nodal anti fuzzy graph. i.e. $\mu(u, v)=c$ for all $(u, v) \in E\left(G_{A}\right)$.

Definition 13. [6] Every vertices and edges in an anti fuzzy graph $G_{A}$ have the unique fuzzy values then $G_{A}$ is called as uninodal anti fuzzy graph. If $\sigma(u)=c_{1}$ and $\mu(u, v)=c_{2}$ in an anti fuzzy graph $G_{A}$ then $G_{A}$ is called as binodal anti fuzzy graph.

Definition 14. The strong neighbourhood of an edge $e_{i}$ in an anti fuzzy graph $G_{A}$ is $N_{s}\left(e_{i}\right)=\left\{e_{j} \in E(G) / e_{j}\right.$ is an effective edge with $\vee N\left(e_{i}\right)$ in $G_{A}$ and adjacent to $\left.e_{i}\right\}$.

Definition 15. An edge $e=\{u, v\}$ of an anti fuzzy graph $G_{A}$ is called an weak edge if $\mu(u, v) \neq \sigma(u) \vee \sigma(v)$.

\section{Total domination on anti fuzzy graph}

In this section, the definition of total dominating set and total domination number on anti fuzzy graph $\mathrm{G}_{A}$ are defined. These concepts are applied on some types of simple anti fuzzy graph $\mathrm{G}_{A}$, few elementary bounds on total domination number are described along with the corresponding theorems and results are illustrated. 
Definition 16. [7] A set $D \subseteq V\left(G_{A}\right)$ is said to be a dominating set of an anti fuzzy graph $G_{A}$ if for every vertex $v \in V\left(G_{A}\right) \backslash D$ there exists $u$ in $D$ such that $v$ is a strong neighborhood of $u$ with $\mu(u, v)=\sigma(u) \vee \sigma(v)$ otherwise it dominates itself.

A dominating set $D$ with minimum number of vertices is called a minimal dominating set if no proper subset of $D$ is a dominating set.

The maximum fuzzy cardinality taken over all minimal dominating set in $G_{A}$ is called a domination number of anti fuzzy graph $G_{A}$ and is denoted by $\gamma\left(G_{A}\right)$ or $\gamma_{A} . i e,|D|_{f}=\sum_{v \in D} \sigma(v)$.

Definition 17. A set $D \subseteq V\left(G_{A}\right)$ is said to be a total dominating set of an anti fuzzy graph $G_{A}$ if for every vertex $v \in V\left(G_{A}\right) \backslash$ $D$ is adjacent to atleast one strong neighbourhood vertex in $D$ and the induced subgraph of $D$ has no isolated vertices. i.e., A dominating set $D$ of an anti fuzzy graph $G_{A}$ is said to be a total dominating set of $G_{A}$ if there exists a vertex in $D$ is not isolate.

A total dominating set $D$ of an anti fuzzy graph $G_{A}$ with minimum number of vertices is called a minimal total dominating set of $G_{A}$ if no proper subset of $D$ is a dominating set.

The maximum fuzzy cardinality taken over all minimal total dominating set is called total domination number of $G_{A}$ and it is denoted by $\gamma_{t}\left(G_{A}\right)$ or $\gamma_{A t}$.

Definition 18. $G_{A}$ is an anti fuzzy graph and $u, v \in V\left(G_{A}\right)$. If $v$ is said to be a support vertex to $u$ then $v$ is adjacent to atleast one end vertex $u$ in $G_{A}$.

Note. Every support vertices in $\mathrm{G}_{A}$ contained in total dominating set of $\mathrm{G}_{A}$. It may dominate more than one vertex in $\mathrm{V} \backslash \mathrm{D}$ and also in $\mathrm{D}$.

\section{Example 1.}

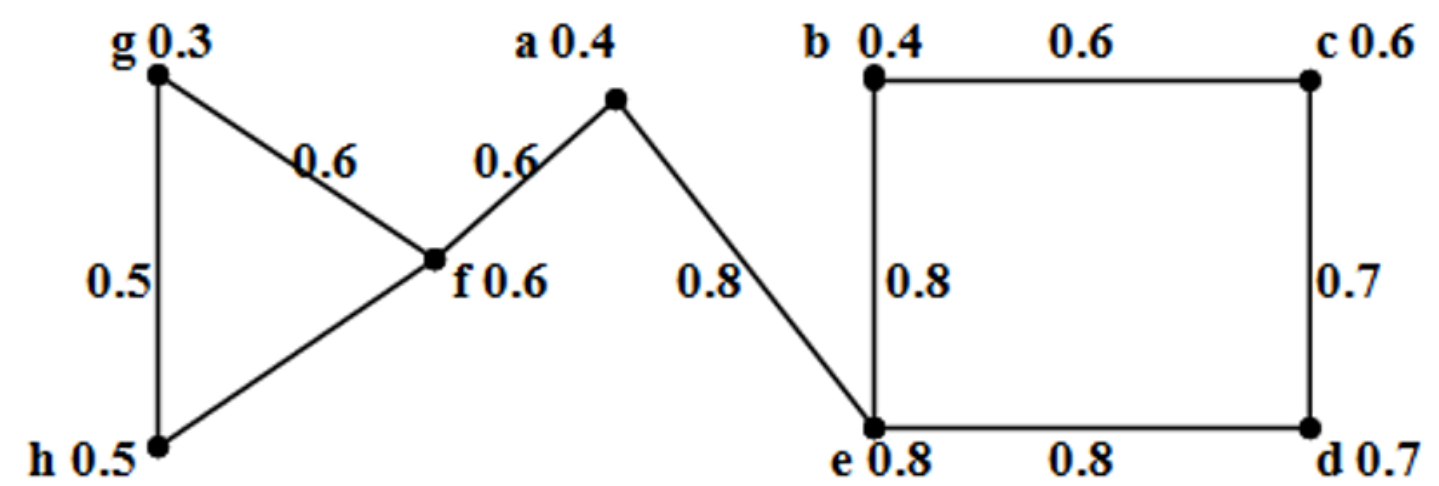

Fig. 1: An Anti Fuzzy Graph $\mathrm{G}_{A}$

In Figure 1, the total dominating sets are $\{\mathrm{d}, \mathrm{e}, \mathrm{f}, \mathrm{g}\},\{\mathrm{d}, \mathrm{e}, \mathrm{f}, \mathrm{h}\}$ and $\{\mathrm{d}, \mathrm{e}, \mathrm{f}, \mathrm{a}\}$. The corresponding total domination numbers are 2.6, 2.3 and 2.4 respectively. Therefore, minimal total dominating set of $\mathrm{G}_{A}$ is $\{\mathrm{d}, \mathrm{e}, \mathrm{f}, \mathrm{g}\}$ and $\gamma_{t}\left(\mathrm{G}_{A}\right)=2.6$ 
Theorem 1. If a total dominating set $D$ is a minimal total dominating set of a connected anti fuzzy graph $G_{A}$ then each $u \in D$ has atleast one the following conditions hold,

(1) There exists a vertex $v \in V \backslash D$ such that $N[v] \cap D=\{u\}$.

(2) $<D \backslash\{v\}>$ contains isolated vertex.

Proof. Let $\mathrm{G}_{A}$ be a simple connected anti fuzzy graph and $\mathrm{D}$ be a total dominating set of $\mathrm{G}_{A}$. Let $\mathrm{u} \in \mathrm{D}$ and $\mathrm{v} \in \mathrm{V} \backslash \mathrm{D}$. consider that $\mathrm{N}(\mathrm{v}) \cap \mathrm{D}=\{\mathrm{u}\}$. Suppose $\mathrm{D}^{\prime}=\mathrm{D} \backslash\{\mathrm{u}\}$ is a total dominating set of $\mathrm{G}_{A}$. Then $\mathrm{v}$ is not dominated by any vertex in $\mathrm{D}$ which is contradict to $\mathrm{D}^{\prime}$ is total dominating set. Thus the vertex $\mathrm{u}$ is only strong neighbourhood to $\mathrm{v}$ and $\mathrm{v}$ is dominated by $\mathrm{u}$. Therefore, $\mathrm{N}[\mathrm{v}] \cap \mathrm{D}=\{\mathrm{u}\}$ and $\mathrm{D}$ is minimal total dominating set. Thus every vertex has atleast one neighbour in D. If $u$ is removed from $D$ then the neighbour of $u$ is isolate in $D$.

Conversely, if the two conditions hold then we prove that $\mathrm{D}$ is a minimal total dominating set of anti fuzzy graph $\mathrm{G}_{A}$. Suppose $\mathrm{D}$ is not minimal total dominating set. Then there exists $u \in D$ such that $D \backslash\{u\}$ is a total dominating set. Thus $\mathrm{u}$ is a strong neighbour to atleast one vertex in $D \backslash\{u\}$. Therefore, $D \backslash\{u\}$ may contains isolated vertex. If $D \backslash\{u\}$ is a total dominating set then every vertex in $V \backslash D$ is a strong neighbour to atleast one vertex in $\mathrm{D} \backslash\{\mathrm{u}\}$. Therefore the second condition fails. Hence $\mathrm{D}$ is a minimal total dominating set.

Proposition 1. For any anti fuzzy graph $G_{A}, \gamma_{t}\left(G_{A}\right) \leq 2 p / 3$ where $G_{A} \neq C_{A}$.

Theorem 2. Let $G_{A}$ be a simple connected anti fuzzy graph and $\bar{G}_{A}$ be an anti complement of $G_{A}$ then $\gamma_{t}\left(G_{A}\right)+\gamma_{t}\left(\bar{G}_{A}\right) \leq$ $5 p / 3$.

Proof. Let us consider that $\mathrm{G}_{A}$ is a simple connected anti fuzzy graph. $\bar{G}_{A}$ may not have any isolated vertices. Then $\gamma_{t}\left(\mathrm{G}_{A}\right)=$ $2 \mathrm{p} / 3$ and $\gamma_{t}\left(\bar{G}_{A}\right) \leq \mathrm{p}$. Therefore, $\gamma_{t}\left(\mathrm{G}_{A}\right)+\gamma_{t}\left(\bar{G}_{A}\right) \leq 2 \mathrm{p} / 3+\mathrm{p}, \gamma_{t}\left(\mathrm{G}_{A}\right)+\gamma_{t}\left(\bar{G}_{A}\right) \leq 5 \mathrm{p} / 3$

Theorem 3. For an uninodal anti fuzzy graph $G_{A}, \gamma_{t}\left(G_{A}\right)=\left\{\begin{array}{l}\leq \frac{p}{2}, \text { for } p \leq q \\ \geq \frac{p}{2}, \text { for } p>q\end{array}\right.$

Proof. Let $\mathrm{G}_{A}$ be an uninodal anti fuzzy graph with order $\mathrm{p}$ and size $\mathrm{q}$.

If $\mathrm{p}=\mathrm{q}$ then the number of vertices and edges in an anti fuzzy graph are equal. Thus every vertex has atleast one strong neighbour in $\mathrm{G}_{A}$. It yields that the vertex may dominate itself or dominate to atleast one vertex in $\mathrm{G}_{A}$. Therefore, $\gamma_{\text {At }} \leq p / 2$.

If $\mathrm{p}<\mathrm{q}$ then the number of vertices is less than the number of edges in an anti fuzzy graph $\mathrm{G}_{A}$ which implies that every vertex is adjacent to more than two vertices in $\mathrm{G}_{A}$. Hence the number of elements in total dominating set is less than or equal to $\mathrm{m} / 2$. Therefore, $\gamma_{A t} \leq p / 2$.

If $\mathrm{p}>\mathrm{q}$ then the vertices have minimum number of neighbourhoods in $\mathrm{G}_{A}$. That is, the vertices are adjacent to atmost $\mathrm{m} / 2$ vertices and not an isolated vertex in $\mathrm{G}_{A}$. Hence the maximum number of vertices dominated itself and gives $\gamma_{A t} \geq p / 2$.

Theorem 4. If $G_{A}$ is an uninodal anti fuzzy graph with no isolated vertices then $\gamma_{t}\left(G_{A}\right)=p-\Delta\left(G_{A}\right)$, where $\Delta\left(G_{A}\right)$ is maximum degree of $G_{A}$.

Proof. Let $\mathrm{G}_{A}$ be an uninodal anti fuzzy graph with no isolated vertices and $\mathrm{D}$ be the total dominating set in $\mathrm{G}_{A}$. Every vertex in D dominates atmost $\Delta(\mathrm{G})$-1 vertices in $\mathrm{V}\left(\mathrm{G}_{A}\right) \backslash \mathrm{D}$ and dominate atleast one vertex in $\mathrm{D}$. 
Hence,

$$
\begin{aligned}
& p=\Delta(G)-1+|D|+1 \\
& p=\Delta(G)+\gamma_{t}\left(G_{A}\right) \\
& \gamma_{t}\left(G_{A}\right)=p-\Delta(G)
\end{aligned}
$$

Theorem 5. For complete uninodal anti fuzzy graph $G_{A}, \gamma_{t}\left(G_{A}\right)=2 \sigma\left(u_{1}\right)$, for all $u_{1} \in V\left(G_{A}\right.$. $)$

Proof. Let $G_{A}$ be a complete uninodal anti fuzzy graph and $\mathrm{D}$ be the total dominating set of $\mathrm{G}_{A}$. By the definition of complete anti fuzzy graph, every pair of vertices are adjacent to each other and by the definition of uninodal anti fuzzy graph, there exists vertices and edges assign the same fuzzy values. In complete uninodal anti fuzzy graph, every single vertex can dominate the remaining vertices. But in total dominating set no vertex is isolated. Hence $D=\left\{\mathrm{u}_{1}, \mathrm{u}_{2}\right\}$.

Therefore, $\gamma_{t}\left(G_{A}\right)=\sigma\left(u_{1}\right)+\sigma\left(u_{2}\right)=\sigma\left(u_{1}\right)+\sigma\left(u_{1}\right)\left\{\right.$ since $G_{A}$ isuninodalantifuzzygraph $\}=2 \sigma\left(u_{1}.\right)$

Theorem 6. For complete uninodal anti fuzzy graph $G_{A}$, and $\bar{G}_{A}$ is a anti complement of $G_{A}$ then $\gamma_{t}\left(G_{A}\right)+\gamma_{t}\left(\bar{G}_{A}\right)=p+2$ $\sigma\left(u_{1}\right)$, for all $u_{1} \in V\left(G_{A}\right)$

Proof. In complete anti fuzzy graph $\mathrm{G}_{A}, \gamma_{t}\left(\mathrm{G}_{A}\right)=2 \sigma \mathrm{u}_{1}$ \{Theorem 5\}, In anti complement of $\overline{G_{A}}$, all vertices are isolated then $\gamma_{t}\left(\bar{G}_{A}\right)=\mathrm{p}$. Therefore, $\gamma_{t}\left(\mathrm{G}_{A}\right)+\gamma_{t}\left(\bar{G}_{A}\right)=\mathrm{p}+2 \sigma\left(\mathrm{u}_{1}.\right)$

Note.

(1) In $\mathrm{G}_{A}$, a pendent vertex incident with only weakest edge then the vertex dominates itself and also it may be a member of total dominating set.

(2) Every vertex in total dominating set may have strong neighbours with effective edge.

(3) $\delta \leq p-\Delta\left(G_{A} \cdot\right)$

Proposition 2. (1) For any v-nodal anti fuzzy graph, $\gamma_{A t} \leq \frac{p}{3}$.

(2) For any e-nodal anti fuzzy graph, $\gamma_{A t} \leq \frac{4 p}{3}$.

Theorem 7. If $G_{A}$ is an anti fuzzy cycle then $\gamma_{A t} \leq \frac{p+q-\Delta\left(G_{A}\right)}{2}$.

Proof. $G_{A}$ is an anti fuzzy cycle then there is a vertex in an anti fuzzy cycle, dominate atmost two neighbours in $G_{A}$. To construct a total dominating set, choose a vertex(say u) which have maximum fuzzy value and this vertex dominate their two neighbours. To make non-isolated vertices in $\mathrm{D}$, choose the strong neighbourhood of vertex u in $V \backslash D$.

Hence $\gamma_{A t} \leq \frac{p+q-\Delta\left(G_{A}\right)}{2}$.

Theorem 8. If $G_{A}$ is an anti fuzzy path then $\gamma_{t}\left(G_{A}\right) \leq p-\delta$, where $\delta$ is minimum degree of $G_{A}$.

Proof. Let $G_{A}$ be an anti fuzzy path with order $\mathrm{p}$ and $\delta$ be the minimum degree of $G_{A}$. Let D be the minimal total dominating set of $\mathrm{G}_{A}$. In an anti fuzzy path, except starting and ending vertices(say $u_{1}$ and $u_{n}$ ) have maximum two neighbours and it may be the set $\mathrm{M}$. Therefore, the total dominating set contains maximum number of vertices from $\mathrm{M}$. The vertices $u_{1}$ and $u_{n}$ dominated by some vertex in M and it contains degree $\delta$. Hence $\gamma_{t}\left(G_{A} \leq p-\delta\right.$.

Theorem 9. Let $G_{A}$ be an anti fuzzy graph and $v \in V\left(G_{A}\right)$ with $\sigma(v)=\wedge \sigma\left(V\left(G_{A}\right)\right)$. If $N_{s}(v)=u$ and $\left|N_{s}(v)\right| \leq 1$ then $u$ belongs to total dominating set of $G_{A}$.

Proof. Let $\mathrm{G}_{A}$ be an anti fuzzy graph and $\mathrm{v} \in \mathrm{V}\left(\mathrm{G}_{A}\right)$ and $\mathrm{D}$ be a total dominating set of $\mathrm{G}_{A}$. Consider that $\mathrm{u} \in \mathrm{V}\left(\mathrm{G}_{A}\right)$ and $\mathrm{N}_{s}(\mathrm{v})=\mathrm{u}$. If $|N(v)| \leq 1$ then $\mathrm{v}$ is adjacent to $\mathrm{u}$ only or it may be isolate in $\mathrm{G}_{A}$. Hence $\mathrm{u} \in \mathrm{D}$. 
Corollary 1. If $\mathrm{G}_{A}$ is an anti fuzzy graph then $\gamma\left(\mathrm{G}_{A}\right) \leq \gamma_{t}\left(\mathrm{G}_{A}\right)$.

Proof. Let $\mathrm{G}_{A}$ be an anti fuzzy graph. Consider that $\mathrm{D}$ is a dominating set and $\mathrm{D}_{t}$ is a total dominating set of $\mathrm{G}_{A}$. Every vertices in $\mathrm{D}$ dominates atleast one vertex in $\mathrm{V} \backslash \mathrm{D}$ also the vertices in $\mathrm{D}$ may be isolated. But by the definition of total dominating set, no vertex is isolated. Therefore the total dominating set contains atleast one vertex is greater than or equal to the dominating set of anti fuzzy graph $\mathrm{G}_{A}$. ie., $|D| \leq\left|\mathrm{D}_{t}\right|$. Hence $\gamma\left(\mathrm{G}_{A}\right) \leq \gamma_{t}\left(\mathrm{G}_{A}\right)$.

Theorem 10. Let an anti fuzzy graph $G_{A}$ contain more than one component without isolated vertices then the total dominating set contains atleast two vertices in each components and $\gamma_{t}\left(G_{A}\right) \leq p$ - $\delta$ where $p=\sum \sigma(u), u \in V\left(G_{A}\right)$ and $\delta=$ $\min \left\{\right.$ minimum degree of $\left.C\left(G_{A}\right)\right\}$

Proof. Let $\mathrm{G}_{A}$ be an anti fuzzy graph and $\mathrm{D}$ be a total dominating set of $\mathrm{G}_{A}$. Consider that $\mathrm{G}_{A}$ contains atleast one component. ie., every component of $\mathrm{G}_{A}$ contains atleast two vertices, Since $\mathrm{G}_{A}$ has no isolated vertices. To construct a total dominating set, there are no isolated vertices in D. Therefore atleast two vertices in each component should be contained in D. Hence $\gamma_{t}\left(\mathrm{G}_{A}\right) \leq \mathrm{p}-\delta$.

Corollary 2. If $\mathrm{G}_{A}$ contains $\mathrm{m}$ components then $\gamma_{t}\left(\mathrm{G}_{A}\right) \leq \sum(\mathrm{p}-\delta)_{i}$, where $\mathrm{i}=1$ to $\mathrm{m}$ components.

Theorem 11. If $G_{A}$ is an anti fuzzy tree then $\gamma_{t}\left(G_{A}\right) \leq p-\sum \sigma\left(u_{i}\right)$, where $u_{i}$ are leaf in $G_{A}$.

Proof. Let $\mathrm{G}_{A}$ be an anti fuzzy tree and $\mathrm{D}$ be a total dominating set of $\mathrm{G}_{A}$. Then the root and subroot vertices of $\mathrm{G}_{A}$ contained in D. Because these vertices have more adjacencies in $\mathrm{G}_{A}$ with maximum fuzzy value which gives except the leaf of a tree remaining all vertices contained in total dominating set. Hence $\gamma_{t}\left(\mathrm{G}_{A}\right) \leq \mathrm{p}-\sum \sigma\left(\mathrm{u}_{i}\right)$

Theorem 12. If $G_{A}$ is star then $\gamma_{t}\left(G_{A}\right)=\sigma(r)+\vee\left(\sigma\left(u_{i}\right)\right)$ where $u_{i}$ is a leaf and $r$ is root of $G_{A}$.

Proof. If $\mathrm{G}_{A}$ is a star and $\mathrm{D}$ is minimal total dominating set of $\mathrm{G}_{A}$. In $\mathrm{G}_{A}$, the root vertex(say $\mathrm{r}$ ) should be adjacent to all remaining vertices in $\mathrm{G}_{A}$ with maximum fuzzy value. Thus it dominates itself and all vertices. Therefore, $D=\{r\}$. But $D$ is a minimal total dominating set. Therefore the vertex $r$ cannot be isolate in D. Hence a support vertex is chosen which has maximum fuzzy value (say $\left.\mathrm{u}_{i}\right)$. Hence $\mathrm{D}=\left\{\mathrm{r}, \mathrm{u}_{i}\right\}$ and $\gamma_{t}\left(\mathrm{G}_{A}\right)=\sigma(\mathrm{r})+\mathrm{V}\left(\sigma\left(\mathrm{u}_{i}\right)\right)$.

\section{Algorithms to find total dominating set of anti Fuzzy graph}

In this section, an algorithm is derived for finding the minimal total dominating set of an anti fuzzy graph $\mathrm{G}_{A}$ using strong adjacency matrix.

Definition 19. [7] An anti fuzzy graph $G_{A}=(\sigma, \mu)$ with the fuzzy relation $\mu$ to be reflexive and symmetric is completely determined by the adjacency fuzzy matrix and it is denoted by $M_{\mu}$. Where $\left(M_{\mu}\right)_{i, j}=\left\{\begin{array}{c}\mu\left(v_{i}, v_{j}\right), \text { for } i \neq j \\ \sigma\left(v_{i}\right), \text { for } i=j\end{array}\right.$ If $\sigma^{*}$ contains $n$ elements then $M_{\mu}$ is a square matrix of order $n$.

Definition 20. [7] Let $G_{A}$ be a simple connected anti fuzzy graph. Then the strong adjacency matrix $\left(M_{\mu}\right)^{\prime}$ is defined as, $\left(M_{\mu}\right)^{\prime}=\left\{\begin{array}{l}\mu\left(v_{i}, v_{j}\right), \text { for } i \neq j \text { and } v_{i} \text { is a strong neighbourhood to } v_{j} \\ \sigma\left(v_{i}\right), \text { for } i=j\end{array}\right.$

Algorithm 1. Let us define the algorithm for finding minimal total dominating set in an anti fuzzy graph $\mathrm{G}_{A}$ with $\mathrm{m}$ vertices. Let $\mathrm{D}$ be a minimal total dominating set of $\mathrm{G}_{A}$.

Step 1: $\quad$ For $\mathrm{G}_{A}$, to construct the $\left(\mathrm{M}_{\mu}\right)^{\prime}$ and $\mathrm{p}=$ sum of main diagonal elements.

Step 2: Let $\mathrm{D}=\emptyset$

Step 3: Find $\sum R\left(u_{i}\right)$ and $\sum C\left(u_{i}\right)$ 
Step 4: $\quad$ In $\left(\mathrm{M}_{\mu}\right)^{\prime}$, choose the maximum value in $\mathrm{R}\left(\mathrm{u}_{i}\right)$ or $\mathrm{C}\left(\mathrm{u}_{i}\right)$. If tie occurs then choose the vertex which have maximum $\sigma\left(\mathrm{u}_{i}\right)$ in main diagonal otherwise break it arbitrarily.

Step 5: Select the corresponding vertex in the selected row as a dominated vertex and write the vertex in the set D.

Step 6: Delete the selected (labeled vertex) row and column with horizontal and vertical line. The resulting reduced matrix is called as $\left(\mathrm{M}_{\mu}\right)^{\prime}{ }_{1}$.

Step 7: If the reduced matrix $\left(\mathrm{M}_{\mu}\right)^{\prime}{ }_{1}$ has any rows and columns goto step 3, until to get only diagonal elements in the reduced matrix.

Step 8: Check the connectivity of the vertices in D. If there exists any isolated vertex then move to step 9. Otherwise STOP.

Step 9: Choose the maximum value in $\sum \mathrm{R}\left(\mathrm{u}_{i}\right)$ from the reduced matrix, check the corresponding vertex is adjacent to the isolate vertex in $\mathrm{V}(\mathrm{D})$ by using $\left(\mathrm{M}_{\mu}\right)^{\prime}$. If the adjacencies between vertices not occur then choose the next maximum value. Repeat this step until D has no isolate vertex.

Example 2. For the Figure 2, The following steps for finding the total dominating set using algorithm are as follows,

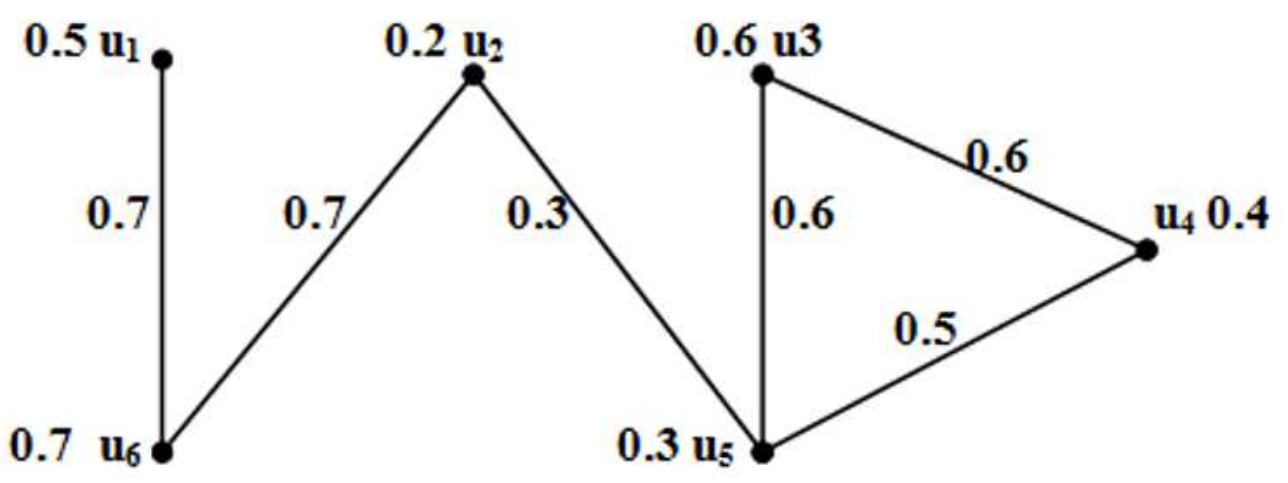

Fig. 2: An Anti Fuzzy Graph $\mathrm{G}_{A}$

Step 1: For the given anti fuzzy graph $\mathrm{G}_{A}$, the strong adjacency matrix $\left(\mathrm{M}_{\mu}\right)^{\prime}$ is given as

$$
\left(\mathrm{M}_{\mu}\right)^{\prime}=\begin{gathered}
u_{1} \\
u_{1} \\
u_{2} \\
u_{3} \\
u_{4} \\
u_{5} \\
u_{6}
\end{gathered}\left[\begin{array}{cccccc}
0.5 & 0 & 0 & 0 & 0 & 0.7 \\
0 & 0.2 & 0 & 0 & 0 & 0.7 \\
0 & 0 & 0.6 & 0.6 & 0.6 & 0 \\
0 & 0 & 0.6 & 0.4 & 0 & 0 \\
0 & 0 & 0.6 & 0 & 0.3 & 0 \\
0.7 & 0.7 & 0 & 0 & 0 & 0.7
\end{array}\right]
$$

Step 2: $\quad$ Let the total dominating set, $D=\emptyset$

Step 3:

$\begin{array}{llllllll}u_{1} & u_{2} & u_{3} & u_{4} & u_{5} & u_{6} & \sum R\left(u_{i}\right)\end{array}$ 


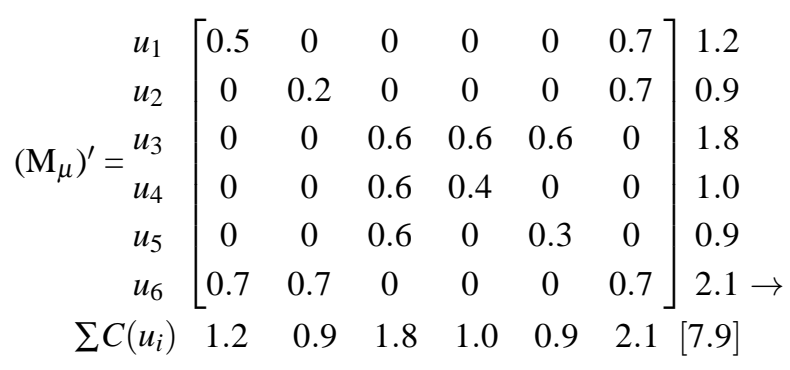

Step 4: $\quad \operatorname{In}(\mathrm{M} \mu)^{\prime}, \mathrm{R}\left(\mathrm{u}_{6}\right)$ has maximum value. Then select $\mathrm{u}_{6}$ and $\mathrm{D}=\left\{\mathrm{u}_{6}\right\}$

Step 5: Delete the selected labeled vertex $\mathrm{u}_{6}$ in row and column wise at $(\mathrm{M} \mu)^{\prime}$. The resulting reduced matrix say $(\mathrm{M} \mu)^{\prime}{ }_{1}$.

Step 6: If $(\mathrm{M} \mu)^{\prime}{ }_{1}$ is a strong adjacency matrix then go to step 3.

Step 3A:

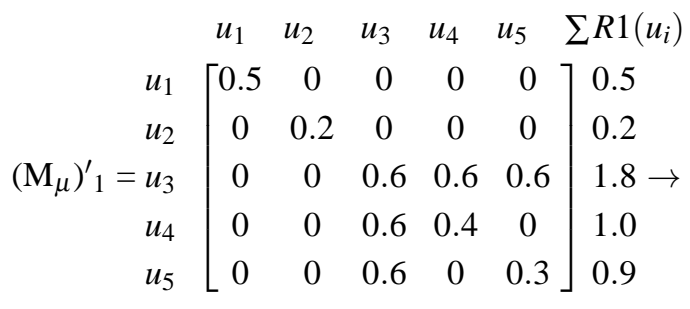

$$
\begin{aligned}
& \Sigma C 1\left(u_{i}\right) \quad 0.5 \quad 0.2 \quad 1.8 \quad 1.0 \quad 0.9[4.4]
\end{aligned}
$$

Step 4A: $\quad$ In $\left(\mathrm{M}_{\mu}\right)^{\prime}{ }_{1}, \mathrm{R} 1\left(\mathrm{u}_{3}\right)$ has maximum value. Then select $\mathrm{u}_{3}$ and $\mathrm{D}=\left\{\mathrm{u}_{6}, \mathrm{u}_{3}\right\}$

Step 5A: Delete the selected labeled vertex $u_{3}$ in row and column wise at $\left(\mathrm{M}_{\mu}\right)^{\prime}{ }_{1}$. The resulting reduced matrix say $\left(\mathrm{M}_{\mu}\right)^{\prime}{ }_{2}$.

Step 7: $\quad\left(\mathrm{M}_{\mu}\right)_{2}^{\prime}$ has four rows with diagonal values only.

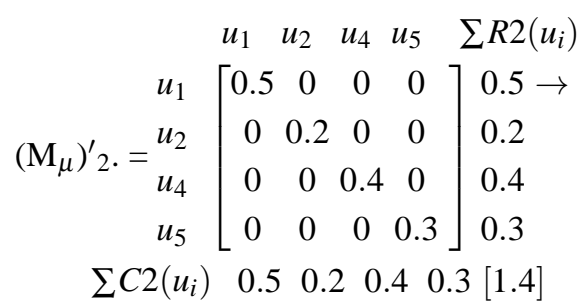

Step 8: Now D has only isolate vertices. In $\left(\mathrm{M}_{\mu}\right)^{\prime}{ }_{2}, \mathrm{R} 2\left(\mathrm{u}_{1}\right)$ has maximum value. From the Figure 2 , it has adjacent with $\mathrm{u}_{6}$ in $\mathrm{D}$. Then select $\mathrm{u}_{1}$ and put in $\mathrm{D}$. then $\mathrm{D}=\left\{\mathrm{u}_{6}, \mathrm{u}_{3}, \mathrm{u}_{1}\right\}$.

Still $\mathrm{D}$ has isolate vertex $\mathrm{u}_{3}$.

Step 8A: $\quad$ In $\left(\mathrm{M}_{\mu}\right)^{\prime}{ }_{2}$, the next maximum value occur at $\mathrm{R} 2\left(\mathrm{u}_{4}\right)$ and it has adjacent with $\mathrm{u}_{3}$ in D. Then select $\mathrm{u}_{4}$ and put in $\mathrm{D}$. Then $\mathrm{D}=\left\{\mathrm{u}_{6}, \mathrm{u}_{3}, \mathrm{u}_{1}, \mathrm{u}_{4}\right\}$.

Now D has no isolate vertex. STOP.

The resulting set $\mathrm{D}=\left\{\mathrm{u}_{6}, \mathrm{u}_{3}, \mathrm{u}_{1}, \mathrm{u}_{4}\right\}$ is a total dominating set of the given anti fuzzy graph $\mathrm{G}_{A}$ and which is also minimal. The total domination number is $\gamma_{A t}=2.2$

\section{Total domination on anti cartesian product of anti fuzzy graphs}

In this section, we apply total domination number on anti cartesian product on same types of anti fuzzy graphs such as cycle, path and complete anti fuzzy graph. To the resulting anti fuzzy graph obtain the bounds on them. In this paper, to

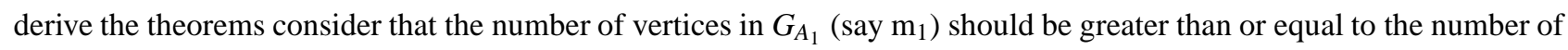
vertices in $G_{A_{2}}$ (say $\mathrm{m}_{2}$ ). 


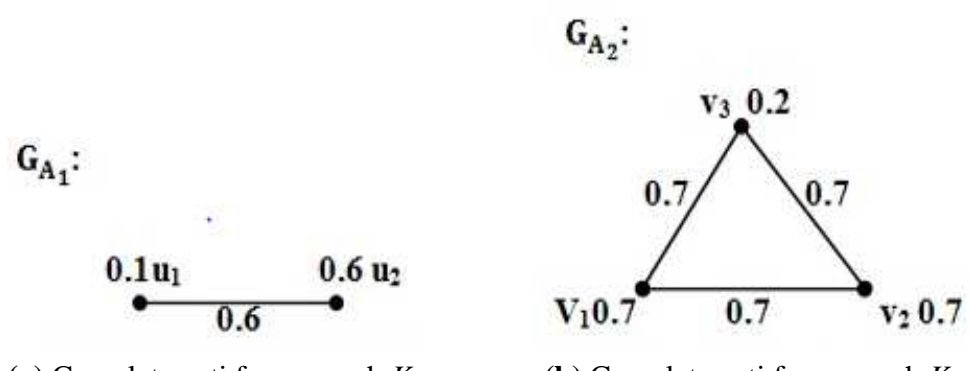

(a) Complete anti fuzzy graph $K_{2}$

(b) Complete anti fuzzy graph $K_{3}$

$$
\mathrm{G}_{\mathrm{A}_{1}} \times \mathrm{G}_{\mathrm{A}_{2}} \text { : }
$$

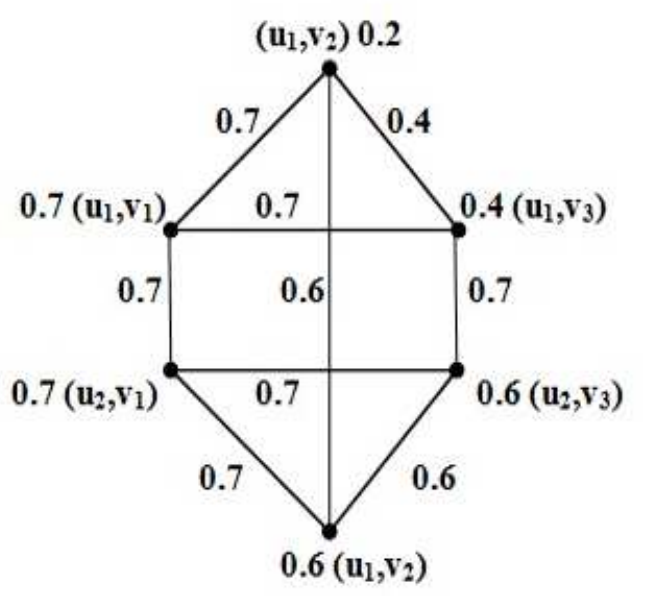

(c) Anti cartesian product of $K_{2} \times K_{3}$

Fig. 3: Anti fuzzy graphs.

Theorem 13.[5] Let $G_{A}$ be an anti cartesian product of anti fuzzy graphs $G_{A_{1}}$ and $G_{A_{2}}$ where $G_{A_{1}}=\left(\sigma_{1}, \mu_{1}\right)$ and $G_{A_{2}}=$ $\left(\sigma_{2}, \mu_{2}\right)$ then $G_{A}=\left(\sigma_{1} \times \sigma_{2}, \mu_{1} \times \mu_{2}\right)$ is an anti fuzzy graph.

Note. In $G_{A}$, the vertices of total dominating set $D$ incident with atleast one effective edges of $G_{A}$ and every vertex in $D$ is a strong neighbourhood to atleast one vertex in $\mathrm{G}_{A}$.

Theorem 14. Let $G_{A_{1}}$ and $G_{A_{2}}$ be any two strong anti fuzzy graphs. $G_{A}=\left(G_{A_{1}} \times G_{A_{2}}\right)$ be an anti cartesian product of anti fuzzy graphs then $\gamma_{t}\left(G_{A}\right) \neq \gamma_{t}\left(\bar{G}_{A}\right)$.

Proof. Let $\mathrm{G}_{A}$ be an anti cartesian product of two strong anti fuzzy graphs $G_{A_{1}}$ and $G_{A_{2}}$. Therefore, there exists no isolated vertices in $\mathrm{G}_{A}$. If each vertex in $\mathrm{G}_{A}$ has minimum number of neighbourhood then $\gamma_{t}\left(\mathrm{G}_{A}\right) \leq \mathrm{p} / 2$. Thus each vertex in $\bar{G}_{A}$ has maximum number of neighbourhood then minimum number of vertices in total dominating set. Hence $\gamma_{t}\left(\mathrm{G}_{A}\right) \neq \gamma_{t}\left(\bar{G}_{A}\right)$.

Proposition 3. Let $G_{A_{1}}$ and $G_{A_{2}}$ be anti fuzzy graphs and $G_{A}$ be the anti cartesian product of anti fuzzy graphs of $G_{A_{1}}$ and $G_{A_{2}}$. Then

(1) $\gamma_{t}\left(G_{A}\right) \neq \gamma_{t}\left(G_{A_{1}}\right) \times \gamma_{t}\left(G_{A_{2}}\right)$

(2) $\gamma_{t}\left(G_{A}\right) \leq\left(\left|V\left(G_{A_{1}}\right)\right| \vee\left|V\left(G_{A_{2}}\right)\right|\right) \times\left(\gamma_{t}\left(G_{A_{1}}\right) \vee \gamma_{t}\left(G_{A_{2}}\right)\right)$.

(3) $\gamma_{t}\left(G_{A_{1}} \times G_{A_{2}}\right) \geq \gamma_{t}\left(G_{A_{1}}\right) \times \gamma_{t}\left(G_{A_{2}}\right)$ 


\section{Example 3. From Figure 3,}

For $G_{A_{1}},\left|V\left(G_{A_{1}}\right)\right|=2, \gamma_{t}\left(G_{A_{1}}\right)=0.7$

For $G_{A_{2}},\left|V\left(G_{A_{2}}\right)\right|=3, \gamma_{t}\left(G_{A_{2}}\right)=1.4$,

For $G_{A_{1}} \times G_{A_{2}},\left|V\left(G_{A_{1}} \times G_{A_{2}}\right)\right|=6 . \gamma_{t}\left(G_{A_{1}} \times G_{A_{2}}\right)=1.4$,

$\left.\left|V\left(G_{A_{1}}\right)\right| \vee\left|V\left(G_{A_{2}}\right)\right|\right) \times\left(\gamma_{t}\left(G_{A_{1}}\right) \vee \gamma_{t}\left(G_{A_{2}}\right)=(2 \vee 3) \times(0.7 \vee 1.4)=3 \times 1.4=4.2 \geq \gamma_{t}\left(G_{A}\right)\{\right.$ proposition3(2)isverified. $\}$

Algorithm 2. For the Figure 3(c), the minimal total dominating set is obtained by using algorithm 1. Applying this algorithm, a strong adjacency matrix for the resulting anti fuzzy graph is constructed.

Step 1: For the given anti fuzzy graph $G_{A_{1}} \times G_{A_{2}}$, the strong adjacency matrix $\left(M_{\mu}\right)^{\prime}$ is given as

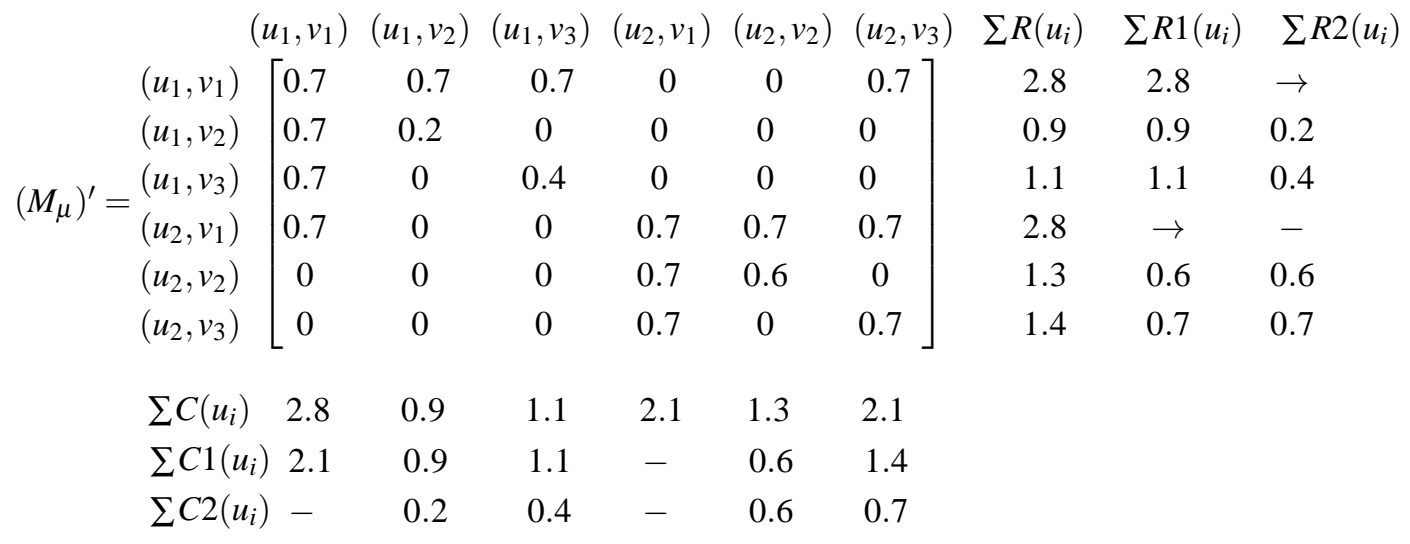

The total dominating set is $D=\left\{\left(u_{1}, v_{1}\right),\left(u_{2}, v_{1}\right)\right\}$. Now D has no isolate vertex. STOP.

The resulting set $D=\left\{\left(u_{1}, v_{1}\right),\left(u_{2}, v_{1}\right)\right\}$ is a total dominating set of the given anti fuzzy graph $G_{A}$ and which is also minimal. The total domination number is $\gamma_{A t}=1.4$.

Proposition 4. Let $G_{A_{1}} \times G_{A_{2}}$ be an anti cartesian product of anti fuzzy paths of $G_{A_{1}}$ and $G_{A_{2}}$. D be the total dominating set of $G_{A_{1}} \times G_{A_{2}}$.

(1) If $\left|V\left(G_{A_{1}} \times G_{A_{2}}\right)\right| \leq 12$ then $G_{A_{1}}$ fiber at $u_{1}$ or $G_{A_{2}}$ fiber at $u_{2}$ contained in $D$.

(2) If $\left|V\left(G_{A_{1}} \times G_{A_{2}}\right)\right|>12$ then atleast two vertices of $G_{A_{1}}$ fiber at $u_{1}$ or $G_{A_{2}}$ fiber at $u_{2}$ is contained in $D$.

Theorem 15. Let $G_{A_{1}} \times G_{A_{2}}$ be an anti cartesian product of anti fuzzy paths of $G_{A_{1}}$ and $G_{A_{2}}$ with $m_{1}$ and $m_{2}$ vertices and order $p_{1}$ and $p_{2}$. D be the total dominating set then $\gamma_{A t} \leq \frac{m_{1} p_{1}+m_{2} p_{2}}{m_{1}+m_{2}}$.

Proof. Let $G_{A_{1}}$ and $G_{A_{2}}$ be an anti fuzzy paths on $\mathrm{m}_{1}$ and $\mathrm{m}_{2}$ vertices with order $\mathrm{p}_{1}$ and $\mathrm{p}_{2}$ and $\mathrm{G}_{A}=G_{A_{1}} \times G_{A_{2}}$ be an anti fuzzy graph on $\mathrm{m}_{1} \mathrm{~m}_{2}$ vertices. Let $\mathrm{D}$ be a minimal total dominating set of $\mathrm{G}_{A}$. The anti cartesian product of anti fuzzy paths looks like a grid graph. By proposition 4 , there exists atleast one vertex in $G_{A_{1}}$ fiber at $\mathrm{u}_{1}$ or $G_{A_{2}}$ fiber at $\mathrm{u}_{2}$. Thus all the vertices in $\mathrm{G}_{A}$ has 3 or 4 neighbours. Hence $\gamma_{A t} \leq \frac{m_{1} p_{1}+m_{2} p_{2}}{m_{1}+m_{2}}$.

Theorem 16. Let $G_{A}$ be an anti cartesian product of anti fuzzy cycles of $G_{A_{1}}$ and $G_{A_{2}}$ with order $p_{1}$ and $p_{2}$. Then $\gamma_{A t} \leq 2$ [ $\left.p_{1}+p_{2}-\delta\left(G_{A}\right)\right]$

Proof. Let $\mathrm{G}_{A}=G_{A_{1}} \times G_{A_{2}}$ be anti cartesian product of anti fuzzy cycles of $G_{A_{1}}$ and $G_{A_{2}}$ with order $\mathrm{p}_{1}$ and $\mathrm{p}_{2}$. Let D be a minimal total dominating set of $\mathrm{G}_{A}$. Every $G_{A_{1}}$ fiber at $\mathrm{u}_{i}$ or $G_{A_{2}}$ fiber at $\mathrm{u}_{i}$ has an anti fuzzy cycle and has atleast two vertices in $\mathrm{D}$ and also all the vertices in $\mathrm{G}_{A}$ has 3 or 4 neighbours. Hence $\gamma_{A t} \leq 2\left[\mathrm{p}_{1}+\mathrm{p}_{2}-\delta\left(\mathrm{G}_{A}\right)\right]$. 
Theorem 17. If $G_{A_{1}}$ and $G_{A_{2}}$ are two complete anti fuzzy graphs on $m_{1}$ and $m_{2}$ vertices with order $p_{1}$ and $p_{2}$. $G_{A_{1}} \times G_{A_{2}}$ is an anti fuzzy graph on $m_{1} m_{2}$ vertices then $\gamma_{t}\left(G_{A_{1}} \times G_{A_{2}}\right)= \begin{cases}p_{2}, & \text { if } m_{1}>m_{2} \\ p_{1}, & \text { if } m_{1}<m_{2}\end{cases}$

Proof. Let $G_{A_{1}}$ and $G_{A_{2}}$ be two complete anti fuzzy graphs on $\mathrm{m}_{1}$ and $\mathrm{m}_{2}$ vertices with order $\mathrm{p}_{1}$ and $\mathrm{p}_{2}$. $\mathrm{G}_{A_{1}}=G_{A_{1}} \times G_{A_{2}}$ is an anti fuzzy graph on $m_{1} m_{2}$ vertices. Let $\mathrm{D}$ be a minimal total dominating set of $\mathrm{G}_{A}$. By the definition of complete anti fuzzy graph every pair of vertices is adjacent to each other. If $\mathrm{m}_{1}>\mathrm{m}_{2}$ then in $\mathrm{G}_{A}=\left(G_{A_{1}} \times G_{A_{2}}\right)$, split the vertex set as $\mathrm{m}_{1}$ component with $\mathrm{m}_{2}$ vertices. That is, each component in $G_{A_{1}} \times G_{A_{2}}$ contain $\mathrm{m}_{2}$ vertices and every vertices in a component is adjacent to each other and the vertex which having the maximum value dominate all the vertices in that component and also it dominate a single vertex in the remaining components also. Similarly, proceed in all $\mathrm{m}_{1}-1$ components. The dominant vertices in each component should be adjacent to each other. i.e., $\gamma_{t}\left(\mathrm{G}_{A}\right)=\mathrm{p}_{2}$. Hence $\gamma_{t}\left(G_{A_{1}} \times\right.$ $\left.G_{A_{2}}\right)=\mathrm{p}_{2}$.

Similarly, if $\mathrm{m}_{1}<\mathrm{m}_{2}$ then $\gamma_{t}\left(G_{A_{1}} \times G_{A_{2}}\right)=\mathrm{p}_{1}$.

\section{Conclusion}

In this paper, total domination number is defined on anti fuzzy graphs. The definition of total domination number is applied on various types of anti fuzzy graph and obtained the bounds on them. An algorithm is described to find the minimal total dominating set for given anti fuzzy graph. The total domination number concept is applied on anti cartesian product of anti fuzzy graphs such as anti fuzzy path, anti fuzzy cycle and complete anti fuzzy graph and obtained the bounds on them and the algorithm is applied on anti cartesian product of anti fuzzy graph to get minimal total dominating set.

\section{Competing interests}

The authors declare that they have no competing interests.

\section{Authors' contributions}

All authors have contributed to all parts of the article. All authors read and approved the final manuscript.

\section{References}

[1] Kaufmann. A., "Introduction to the theory of Fuzzy Subsets", Academic Press, Newyork (1975).

[2] Mordeson. J.N., and Nair. P.S., " Fuzzy graphs and Fuzzy Hypergraphs", Physica Verlag, Heidelberg, 1998; second edition 2001.

[3] Mordeson. J.N., and Peng. C.S., "Operations on fuzzy graphs”, Information Sciences, vol. 79 (1994), pp. 159-170.

[4] Muthuraj. R., and Sasireka. A., "On Anti fuzzy graphs”, Advances in Fuzzy Mathematics, Vol. 12, No.5 (2017), pp. 1123 - 1135.

[5] Muthuraj. R., and Sasireka. A., "Some Characterization on Operations of Anti Fuzzy Graphs", International Conference on Mathematical Impacts in Science And Technology, (MIST -17), November 2017, IJRASET, pp. 109-117.

[6] Muthuraj. R., and Sasireka. A., "Some Types of Nodal and Edge Regular Anti Fuzzy Graph", international journal of Fuzzy Mathematical Archive, Vol.14, No. 2, 2017, pp. 365-378.

[7] Muthuraj. R., and Sasireka. A., " Domination on Anti Fuzzy Graph”, international journal of Mathematical Archive, Vol.9, No. 5, 2018, pp. 82-92. 
[8] Rosenfeld A., Fuzzy graphs. In: Zadeh, L.A., Fu, K.S., Shimura, M (Eds.), Fuzzy Sets and their Applications, Academic Press, New York, (1975).

[9] Seethalakshmi. R., and Gnanajothi. R.B., "Operations on Anti fuzzy graphs", Mathematical Sciences International Research Journal, Vol 5,Issue 2 (2016), pp. 210-214.

[10] Somadsundaram. A., and Somadsundaram. S., ” Domination in fuzzy graphs”, Pattern Recognit. Let., 19(9)(1998),pp.787-791.

[11] Zadeh. L.A., "Fuzzy sets", Information Sciences, No. 8 (1965), pp. 338-353. 